\title{
Enabling relationship building in tabletop-supported advisory settings
}

\author{
Heinrich, Peter ; Kilic, Mehmet ; Aschoff, Felix-Robinson ; Schwabe, Gerhard
}

\begin{abstract}
Recent research has shown that financial advisory encounters can successfully be supported with ITartifacts. Tabletop scenarios, for example, can increase the transparency of the advisory process for customers. However, we have also had the experience that the relationship quality as experienced by customers can suffer severely when IT-artifacts are introduced. Based on these experiences, we developed guidelines for both, the artifact- design itself as well as for the environment in order to avoid this effect, and implemented them in one of our prototypes. The evaluation reveals that these measures proved to be effective. With the reported study, we seek to enhance our design knowledge of IT-supported advisory scenarios with a special focus on relationship building. In a larger context, we argue that the use of IT during sensitive face-to-face encounters will be of growing significance in the future but, as yet, is hardly understood. We make a contribution in this area with our generic requirements, design principles and evaluation.
\end{abstract}

DOI: https://doi.org/10.1145/2531602.2531697

Posted at the Zurich Open Repository and Archive, University of Zurich

ZORA URL: https://doi.org/10.5167/uzh-94020

Conference or Workshop Item

Published Version

Originally published at:

Heinrich, Peter; Kilic, Mehmet; Aschoff, Felix-Robinson; Schwabe, Gerhard (2014). Enabling relationship building in tabletop-supported advisory settings. In: 17th ACM Conference on Computer Supported Cooperative Work Social Computing, Baltimore, MD, 15 February 2014 - 19 February 2014. ACM, 171-183.

DOI: https://doi.org/10.1145/2531602.2531697 


\title{
Enabling Relationship Building in Tabletop-supported Advisory Settings
}

\author{
Peter Heinrich, Mehmet Kilic, Felix-Robinson Aschoff, Gerhard Schwabe \\ University of Zurich, Department of Informatics \\ Binzmühlestrasse 14, 8050 Zurich, Switzerland \\ \{peterhe,kilic,aschoff,schwabe\}@ifi.uzh.ch
}

\begin{abstract}
Recent research has shown that financial advisory encounters can successfully be supported with IT-artifacts. Tabletop scenarios, for example, can increase the transparency of the advisory process for customers. However, we have also had the experience that the relationship quality as experienced by customers can suffer severely when IT-artifacts are introduced. Based on these experiences, we developed guidelines for both, the artifactdesign itself as well as for the environment in order to avoid this effect, and implemented them in one of our prototypes. The evaluation reveals that these measures proved to be effective. With the reported study, we seek to enhance our design knowledge of IT-supported advisory scenarios with a special focus on relationship building. In a larger context, we argue that the use of IT during sensitive face-to-face encounters will be of growing significance in the future but, as yet, is hardly understood. We make a contribution in this area with our generic requirements, design principles and evaluation.
\end{abstract}

\section{Author Keywords \\ Tabletop, Relationship Building, Advisory Scenario}

\section{ACM Classification Keywords}

Design, Human Factors, Economics

\section{1}

\section{INTRODUCTION}

An advisory encounter is a crucial social process often taking place between the representative of an institution and a client. There are a number of different conceptualizations of the advisory process (see [25] for a discussion of different perspectives). In a general sense, one can describe an advisory process as the interaction between two persons, where one supports the other in solving certain problems for reaching certain decisions. Advisory encounters are interaction scenarios that have been known for a long time in the areas of medicine, law or sale.

Permission to make digital or hard copies of all or part of this work for personal or classroom use is granted without fee provided that copies are not made or distributed for profit or commercial advantage and that copies bear this notice and the full citation on the first page. Copyrights for components of this work owned by others than ACM must be honored. Abstracting with credit is permitted. To copy otherwise, or republish, to post on servers or to redistribute to lists, requires prior specific permission and/or a fee. Request permissions from Permissions@acm.org.

CSCW'14, February 15 - 19 2014, Baltimore, MD, USA

Copyright 2014 ACM 978-1-4503-2540-0/14/02 ...\$15.00.

http://dx.doi.org/10.1145/2531602.2531697
Recent research has demonstrated that these kinds of advisory scenarios can successfully be supported by ITartifacts [19,25]. For example, Nussbaumer et al. [20] conducted a number of experimental tests in which the perceived transparency of a financial advisory process could eventually be increased. These experiments, however, showed that the quality of the information that is exchanged between the advisor and the client is not the only crucial factor; rather, the relationship between the client and the advisor can considerably suffer because of the introduced IT-artifact.

This is especially problematic because the quality of the relationship is a crucial factor for the advisory process. According to Jungermann [12], the social dimension of these interactions is at least as important as the information that is exchanged between the client and the advisor.

The crucial aspect of IT influence on relationship building in such sensitive face-to-face advisory scenarios has hardly been addressed by previous researchers. In addition, we lack empirically-founded design knowledge how these scenarios are to be conceptualized and implemented. This holds especially for the emerging technology of tabletops which are expected to change the advisory setting in many areas. Accordingly, we pursue the following research question: How can we enable relationship building in a tabletop-supported advisory setting?

By answering this question, we contribute to the design knowledge on sensitive face-to-face scenarios that are crucial in many areas such as financial transactions, legal advice and health issues. Based on the experiences of the evaluations in the financial advisory area, we develop a number of generic requirements and design principles to support relationship building between an advisor and a client. In this paper, we further demonstrate, how these design requirements and principles can be implemented in a tabletop supported advisory encounter. In an analysis of the time, spent for relationship building (mutual face gaze) we compare two tabletop systems: Prototype 1 that does not implement our design requirements and principles and Prototype 2 that does. The evaluation reveals that our guidelines prove to be effective. 
Popular lore claims that there is no second chance to make a first impression. Indeed advisory scenarios are sensitive social interactions which can set the tone for the interactions to come. This is especially the case if the two parties meet only a few times or only once. The role of relationship has been intensely researched in the background of services selling [5] and e-Commerce [22]. Results from that research indicate that the relationship between seller and client is crucial for future sale interaction.

In our research, we focus especially on face-to-face advisory encounters in the financial area. These situations are characterized by the fact that the advisor and the client meet only a few times. Thus, the advisor does not have much time to win the respect and trust of the client. Misunderstandings, irritations or a negative personal atmosphere can hardly be corrected.

In this context, by relationship building we refer to the establishment of a trustful connection in which the client feels taken seriously, having his needs attended to and being treated respectfully. Overall the client should feel comfortable to reveal information that is important for the solution or decision making process.

In such a setting, establishing trust is key to a successful collaborative service encounter [12]. Advisors are trained to establish and maintain a personal relationship, and, for this reason, have thus long resisted using any technology during the actual service encounter, as they are afraid of interference [26].

\subsection{The role of IT-artifacts in advisory encounters}

Advisory services are normally performed in a structured way. In most cases, an investment advisory service will include the following steps: understanding the customer's situation and needs, analyzing her risk preferences and capability, proposing a strategic asset allocation to different asset classes (such as shares or bonds) and selecting specific products (c.f. [12] for an explicit phase model).

Although Stewart et al. [32] proposed collaborative interfaces for face-to-face sales-oriented collaboration, few studies have been undertaken, despite a rising interest in the marketing literature. There, the concept of value co-creation proposes a joint configuration as solution [23].

Apart from that, literature offers several good reasons to support these encounters with appropriate IT-artifacts:

- It has been shown that IT-artifacts can enable the customer to actively participate in the creation of a solution matching his problems [25].
- The IT-system can make the encounter more transparent by sharing information between client and advisor with the help of a shared artifact [20].

- (IT-)Artifacts can support both clients and advisors in the process of "common objectification" [35]. Weber et al. [35] describes common objectification as the act of sharing individual knowledge and expertise through materialized items created by the group members. An IT-system can provide such a shared information space to support this task.

- By providing a virtual form of reality, IT-artifacts can also foster efficient knowledge transfer by enabling situated learning in general [10] and also within the advisory encounter [9].

- IT-artifacts that are used by both advisor as well as client can be a valuable tool to document important information for later advisory sessions or services $[\mathrm{WpHG}]^{1}$.

- IT-artifacts like tabletop systems (in contrast to other display types) can help to seamlessly integrate traditional use of paper (e.g., proposed by [2,31]), which might still be required in such settings.

IT usage, however, also has its downsides. While necessary to achieve goals effectively, IT usage does consume precious time that could be spent otherwise in interpersonal communication. Thus, the more heavily these tools are used in the advisory situation, the less time there is available for relationship building.

\subsection{Previous research in supporting face-to-face interactions with regard to relationship building}

The connection between working with an artifact, as well as establishing and maintaining a personal relationship, has been an important design issue in $\mathrm{CSCW}$ research for more than two decades: "Successful technological augmentation of a task or process depends upon a delicate balance between good social processes and procedures with appropriately structured technology" [6]. Very early on, the importance of maintaining eye contact was a key challenge for collaborative rooms [15]. In those days embedded solutions connected single-user computers into a physical conferencing table. Working with these multi-user interfaces turned out to be challenging, as users had to align the actions of others into their own mental models. In consequence, systems were designed for aligning artifact related work with eye contact during distributed collaboration $[16,18]$. The importance of eye contact for interpersonal relations is also reflected by the literature from psychology: Research shows that eye contact can

\footnotetext{
${ }^{1}$ WpHG: $§ 34$ German Securities Trading Act
} 
influence the interplay between two persons on various levels. For example, eye contact (see [13] for a comprehensive summary on this research) indicates whether a person is paying attention to a certain situation, whether a person is perceived to be competent (more eye contacts are associated with more competence) and whether a person is perceived to be credible (more eye contacts are associated with more credibility). In addition, eye contact is generally perceived as an indicator of how much a person is attracted to another person.

Furthermore, eye contact serves as an important coordination function for turn taking in communication. For example, a speaker may hold a longer gaze at a person to indicate that he/she is done speaking and that it is the other person's turn to speak. This indicates that an IT-artifact can alter or disturb a communicational setting on a relatively subtle level. The participants might then develop a feeling of discomfort or confusion without really being able to describe the cause of this problem.

With the advent of large interactive displays, researchers started to focus on face-to-face interactions with "single display groupware" [32]. Using a single display reduced the cognitive effort of synchronous collaboration, as all participants could directly observe the actions of their collaborators. But not all display types seam to work equally well in service encounter settings: Regarding standard PC-systems, Novak et al. [19] have shown that a service encounter, supported by a standard PC-monitor, can even worsen the perceived information asymmetry. Regarding large displays, Rogers et al. [24] found, that horizontal oriented surfaces (like tabletop systems) foster cohesive group work far better, than vertical displays or standard PC-monitors. Tabletop environments, have further often been used in other group settings as a single shared artifact and been credited for their ease with interpersonal communication [8]. Researchers reported a significant increase in eye contact in contrast to using a desktop monitor as single display interface [11]. Further, a tabletop system closely resembles the known working environment to which clients and advisors are used to.

Tabletop computers can also ease aligning artifact manipulation and non-verbal communication [33]. The increasing sophistication of tabletop computers appears to be the solution for Scott et al.'s [27] design guideline: "Technology that provides little or no overhead to performing or switching between activities would allow users to transition easily between activities, focusing instead on communication." However, as we will show in this paper, the introduction of tabletop systems alone is not sufficient to realize successful relationship building. Further requirements need to be considered.

For tabletop activities in general, people favor to sit opposite to each other [27,30], and thus could establish eye contact by just lifting their heads and/or their eyes. Despite some advances of a face-to-face seating arrangement [17], the orientation of written information on tabletop computers remains an unsolved problem when users sit on opposing sides of the table. Thus, in recent publications [28,33], the settings comprise more people sitting in a 90 degree angle or beside one another when participants want to collaborate and use written information. This is also in line with the research of Wallace et al. [34], that describes that an adjacent (90 degree angle) seating arrangement is preferred over a face-to-face seating arrangement when working collaboratively. This seating arrangement appears to be a good compromise to support comprehension, coordination and ownership of objects [14]. This aspect is also related to the research on proxemic interaction and (IT-) artifact use as, for example, presented by Ballendat [1]. Proxemics describes how people interpret spatial distances to mediate relationships to other people and objects [7]. This line of research underlines the importance of environmental variables for the interaction between persons and between persons and artifacts. Up to now, this research, has been directed at larger changes in body movement, for example, when a person walks and her interpersonal distance to other persons or objects is changing (e.g. from the "public" distance to the "personal" distance). The aspects of body position we are focusing, on is on a smaller and more subtle scale. We will show how small modifications of body and head position in a face-to-face situation can influence the relationship between these two persons.

Based on this background, we do not limit our study to the user interface (UI) only. While being a central element of the interaction, the UI is only one component that influences the behavior of clients and advisors. One also needs to consider additional aspects, such as body positions or the questions what users do when they are not interacting with the artifact [1]. Thus, our analysis will target not only the UI but the advisory setting in general.

\section{NAIVE DESIGN AND EVALUATION OF PROTOTYPE 1}

In 2010, we started a joint research project with a major Swiss bank on improving their investment advisory service for their affluent customer segment (50'000 - 500'000 CHF). In the course of this collaboration, Prototype 1 was developed and tested. The main goal of this prototype was to improve the quality of the advisory process. One crucial aspect in this context was the transparency of the process and the exchanged information [20].

We used a tabletop system to provide a shared artifact workspace [25]. The prototype was designed to support financial advisory encounters within the property of the bank. Since it was not our primary goal to remove all forms of paper from the advisory process but to have it co-existing with an IT-artifact, a tabletop system provided a reasonable working environment that supported both styles at the same time. Note-taking especially was an example of key use of 
paper in this scenario. A tight and seamless integration (as mentioned in section 2.1) seamed not necessary for the purpose of note-taking.

The prototypes and test scenarios described in the following sections have also been published in Nussbaumer et al. $[20,21]$ when researching the transparency of the process. In this paper, we focus on aspects of relationship building in these experimental scenarios that have not been published previously. To this end, we draw on additional data analysis and we explicate the design requirements and the design principles that were established with regard to the relationship dimension.

User Interface: The prototype was built on a Microsoft Surface table, intended to be used as a single shared artifact by both client and advisor. The user interface design of Prototype 1 guided the clients and advisors directly through a suggested advisory process. Each step of the advisory process (e.g., Personal Data, Risk Analysis, Financial Strategy, etc.) was supported by a dedicated screen. On top of the screen there was a navigable process map indicating the current step in the context of all the other steps. We call this the "slide-metaphor," as the visualization is similar to the way information is presented in PowerPoint. Figure 2 illustrates the visualization and interaction possibilities while defining an asset strategy. For a full view of the ITsupported advisory environment see Figure 6.

Body Position: During the advisory sessions, the client sat at the long end of the surface table, with the advisor sitting at the short end. The screen was oriented towards the client, i.e., the client could read texts without effort while the advisor had to mentally rotate the text 90 degrees (Figure 1).

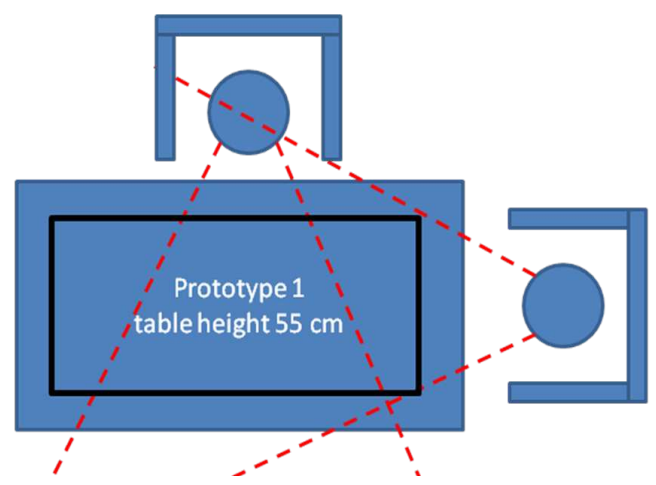

Figure 1: Seating arrangement of Prototype 1 (corner seating).

Evaluation: The prototype was evaluated in a realistic setting with four experienced financial advisors from a major Swiss bank and 12 test-clients. The tested scenario was that of a prospective customer. In the scenario they had to decide on an investment strategy to invest the fictional amount of about US\$250,000. All test-clients received two treatments: one traditional pen and paper advisory and one IT-supported advisory encounter [21]. Half of the participants started with IT-supported advisory service, while the other half started with the traditional setting.

The IT-supported sessions were recorded on video. To compare the two experimental conditions, we conducted interviews in which we asked the participants about their experiences with the IT-supported and the traditional advisory scenarios. We analyzed the recordings of these interviews for all aspects of relationship building as well as for those factors that might affect this relationship building.

Results: The analysis of the interviews revealed that many participants referred to the relationship aspect of the situation. Out of 12 participants, ten participants mentioned this aspect in some form. They pointed out that they were too focused on the artifact, extremely distracted, and that the interpersonal contact without the artifact was more pleasant. One participant described this as: "I felt that I'm not in the focus, but the computer is"; another one pointed out, "It's like a triangle relationship, the advisor and table on one side and me on the other side."

Additional feedback included: "The traditional setting was more personal; in the IT-supported setting it felt like the table was in the focus not the advisory service." "During the IT setting, the personal aspect was lacking; everything went very quick, it would be better to somehow use it (the system) cooperatively." "The computer appears to be in between." "The most annoying was that he (the advisor) looked at the artifact, instead of looking at me."

In addition to this aspect, half of the participants also commented on aspects of distraction. Here, the participants described that there was so much information that they felt overwhelmed and were overly fascinated by the artifact. One participant reported: "You have to wait a while before you can say something." A second one felt overloaded with information coming from two sources: "A lot of information from the table, and the advisor and I have to handle this." Some also felt inhibited, stating: "One was so fascinated by the table that one did not ask questions." Finally, the surveys revealed that the participants preferred the traditional setting to the artifact-supported setting [21]. 


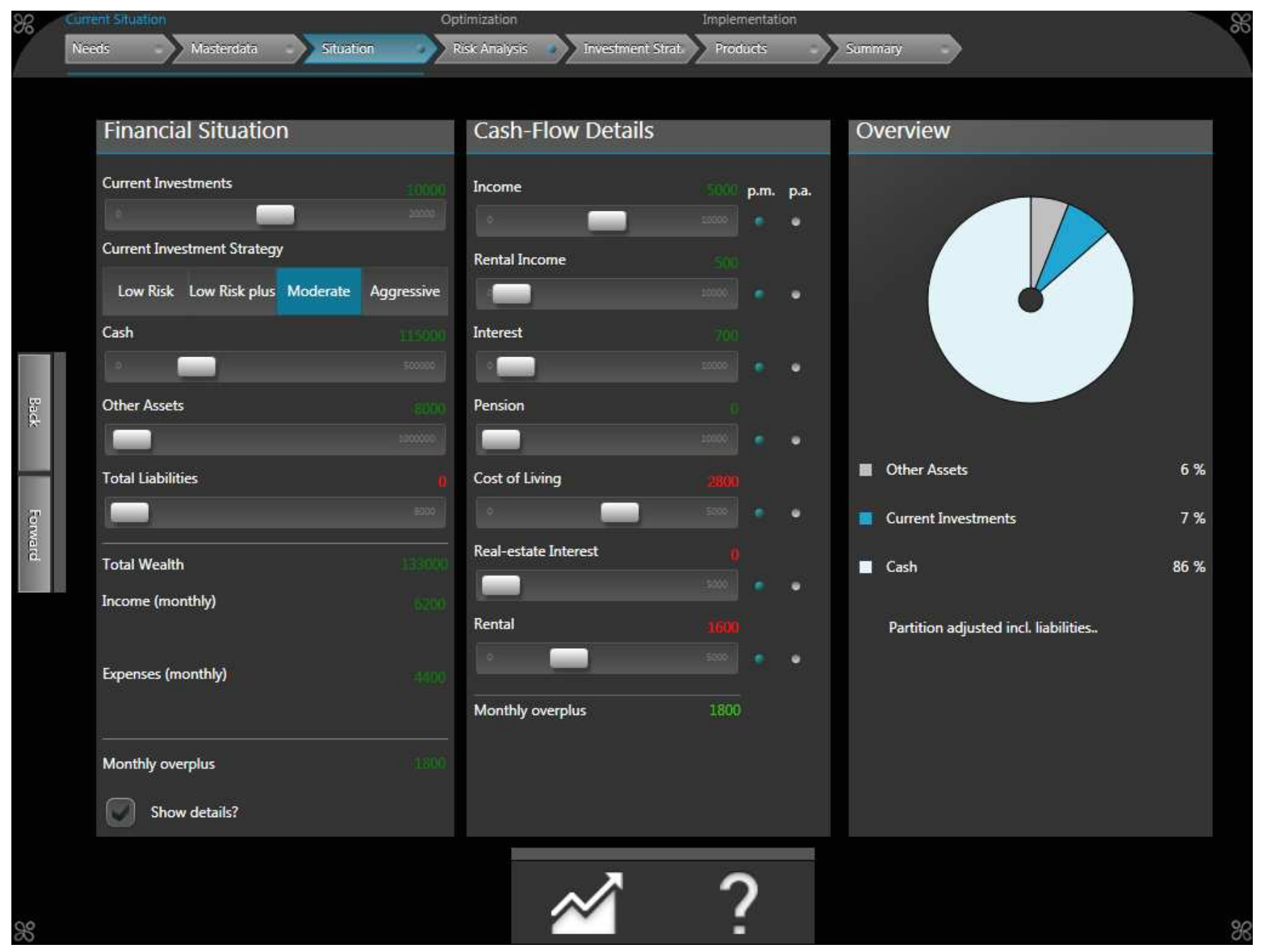

Figure 2: "Slide-metaphor" of the Prototype 1 (full extent of tabletop display, original interface was in German language).

\section{4 \\ PROBLEM IDENTIFICATION AND GENERIC REQUIREMENTS}

The literature [8] describes numerous settings in which partners successfully collaborate while using shared artifacts on table-top computers. In our first evaluation, however, this did not seem to be the case. Based on the evaluation described in the previous section, we conclude that the scenario described for Prototype 1 does not lead to a functioning relationship between advisor and client in many cases. As seen in the evaluation, many participants accredited the presence of the IT-artifact for the disturbed interpersonal relationship. Therefore, our main solution objective is:

Solution Objective: Establish effortless relationship building in IT supported face-to-face advisory encounters.
With "effortless" we mean that it did not take the participants noticeable effort, i.e., more effort than s/he was used to from similar unsupported situations.

Similar to the work of Haller et al. ("Communication space" and "Task space") [8], we introduce the notion of spaces: "relationship building space" (RBS) and "artifact work space" (AWS) (Figure 3). Both RBS and AWS are physical spaces persons can use in their sole discretion. The AWS is defined as the space the persons look at if they want to work with the artifact. RBS on the other hand is defined as the space the persons look at when they want to engage in relationship building. The RBS therefore resembles the space where relationship building is possible from an external point of view.

However, humans are only able to focus on a single point in space at any given time. Thus, we end up with a mutually exclusive three state model of each participant being in one of: 1) in an artifact state (the person is focused on the artifact itself); 2) in a relationship state (the focus is on the 
other person, probably seeking eye contact), or 3), the person is looking somewhere else.

To visualize these states and transitions, we developed a state model (Figure 3) for single or two person situations. This model describes the situation where one participant wants to change its state from being in AWS to RBS. As discussed in the literature section, people feel the need to engage in the RBS, for example, to signal a misunderstanding [13], to ground their communication [3] or to assess the personality of the other person. We assume relationship building to be efficient when both persons are in the RBS state at the same time.

In the discussion following, we focus on the transitions leaving the AWS states. We will argue under which circumstances people can enter RBS directly via transition "A" or why they fail and take transition " $\mathrm{B}$ " or " $\mathrm{C}$ " instead.

Single person

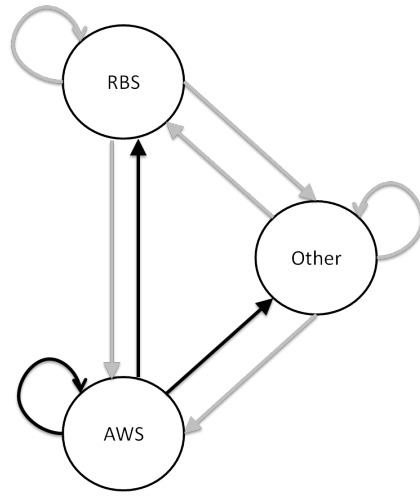

Figure 3: States of focus (left: single person; right: combined model for advisor and client).

The failed relationship building with Prototype 1 implies that the participants did not reach the RBS state often enough or did not stay there long enough.

For a successful transition into the RBS state, three things are necessary: First, both participants have to be aware of the existence of that state. Second, to be successful, the transition into the RBS-state has to be a coordinated transition, leading to both participants being in the RBS simultaneously (Transition A, Figure 3). Third, the affordance to transit into the RBS-state has to be low enough for the participants to switch deliberately into the RBS state.

The AWS state is inherently created with provision of the artifact to its users. However, the relationship building space does not seem to instantiate itself automatically. In order to ensure successful relationship building between client and advisor, that space has to be created and maintained throughout the session. Relationship building is not a one-time effort but rather an ongoing procedure running in parallel to the technical task of problem solving and decision making. This implies constant switching between the two spaces.

To motivate people to engage in the relationship building space, they have to be accommodated to it. In many non-ITsupported situations this happens intuitively when two persons interact with one another. However, it seems that this step is easily omitted when people are confronted with an attractive IT-artifact. Lacking such a relationship can hamper any further development of the relationship when using an IT-artifact right from the beginning. The first encounter in such a setting is probably crucial and sets the stage for the relationship that is established between the two. If the RBS is not introduced at the very beginning of the session, the advisor and client lack common ground [3].

Generic Requirement 1: Initially accustom the participants to the relationship building space and instantiate a basic relationship.

While interacting with Prototype 1, participants complained about the process the IT-artifact imposed on the interaction between themselves and the advisor. One participant stated: "The process was predefined and it was difficult to ask questions." Another one said: "It should not be like an assembly line - it should not be that standardized." One participant suggested that there should be a period of talking to the advisor before the IT-artifact was introduced.

Even though it was not the intention at the time of development, we enabled the experience that participants as well as advisors followed the pre-given structure of the interface very strictly. As the visualized process had no relationship building related activities, they were simply omitted by the participants. One experience was that a process visualized by IT could have a much stronger effect on the socializing behavior of participants than was intended by the developers. While the process was meant rather to help participants not to forget anything important and to provide some orientation, the participants seemed to interpret the process as an instruction for behavior. Applied to our state model, instead of switching into the relationship building state, they stayed in the AWS state (transition "B", Figure 3 ). In contrast to transition $A$ and $C$, transition $B$ was explicitly expressed and visualized within the artifact design itself.

This led to the impression that personal or social aspects do not have any place in the advisory process. This aspect conflicts with the guidelines proposed by Ruth Cohn [4] for her Theme-centered Interaction Approach: Disruptions have priority, meaning that it does not make sense to continue with a process if there is conflict on a behavioral level. Thus, we formulate the following requirement:

Generic Requirement 2: Avoid UI designs that bind participants to the artifact space due to a prescribed process. 
In the first setting, the participants were sitting in a $90^{\circ}$ orientation to each other. Due to the low table height, they had to bend a little over the artifact. To switch between the AWS and RBS, they had to at least rotate their heads towards each other. To get into a relaxed body position, they had to lean back and thus move their entire upper body.

We assume that these high switching costs hampered the transitions into the RBS. The participants referred to this issue, stating that the situation with the table was not very comfortable. One participant said: "It was disturbing because it [the table] was too low; I had to bend my head over it." Another one would even prefer to stand. To address this issue, we formulated the design requirement:

Generic Requirement 3: The physical effort to switch into the relationship building space (RBS) has to be low. Avoid the need for body movement at all.

Another cause we assume to play a role is that a high cognitive effort can also reduce the ability to grasp the other person's state. We arrived at this conclusion after hearing the remarks of participants. A number of participants complained that they "had to concentrate too much on the tabletop," that "the advisor had to concentrate too much on the tabletop" or that "it was difficult to process the information and relate to the advisor at the same time."

As the interviews reveal, the participants were aware of their own cognitive effort required and the resulting lack of time to personally interact with the advisor. We assume that the constantly changing screen forced clients into building a new mental model for each screen - probably also trying to keep in mind the connection to the other hidden screens. This led to the effect that their focus was glued to the artifact space in a (subconscious) fear that they might miss something or that they would not be able to orient themselves again after returning from the relationship space. To address the cause, we formulated the design requirement:

Generic Requirement 4: The cognitive effort of leaving the artifact space has to be low.

If one person is unaware of the other person's intention to switch into RBS, coordinated state switches are impossible or severely hampered at best. Humans only have a limited peripheral field of vision and a very limited area of focus. The advisor seeking eye contact with the client is probably one of the most important indicators that the advisor is seeking a connection with the client. The client, on the other hand, is irritated when he feels the need for attention when the advisor is engaged with the artifact.

The seating and body position in the first setting did not allow them to focus on the artifact while being able to track the other person's point of focus. The face of the advisor, for example, simply vanished out of the peripheral field of vision when the customer had to look at the center of the artifact. Thus, coordination might have been difficult, often resulting in uncoordinated space switches (transition "C", Figure 3). Therefore we propose:

Generic Requirement 5: Ensure effortless sensing of each other's space switches, utilizing the peripheral field of vision.

\section{DESIGN PRINCIPLES AND THEIR IMPLEMENTATION IN PROTOTYPE 2}

\section{Creating and maintaining the relationship building space:}

To accommodate the person to the $R B S$, we suggest introducing a dedicated small talk phase prior to the artifact related work itself. In addition to accommodating the person to the space, the small talk phase is used to establish a first relationship between advisor and client. Thus, this intervention has actually two goals: Firstly, to make participants aware that a relationship building space exists and that it is accepted or even desired within the "interaction protocol" to switch into this state. Secondly, the two participants establish an initial relationship that makes it more likely for them to return to the relationship building state. This initial relationship should also lead to an interaction in which later disturbances (e.g. a short lack of eye contact) are not experienced as severe by the participants.

Design-Principle 1 (to address generic requirement 1): Establish a basic relationship in IT-supported advisory sessions with a dedicated small talk phase at the beginning.

Implementation: For the Prototype 2 evaluation, the advisors were instructed to be seated at the multi-touchtable but to disregard the artifact during the initial small talk phase; rather they should use a physical notepad and a pen to write down useful information during that phase, exactly what they were used to using in the traditional setting.

To overcome the interpretation of the rigid process visualization that no space was available to the relationship building activities, we suggest omitting any direct visual representation of the process.

Design-Principle 2 (to address generic requirement 2): Omit any visualizations of a determined process and avoid any cue to the next activity in AWS.

Implementation: To implement the described visual representation, we mapped each activity to a dedicated widget (software tool supporting the activity). All widgets were freely movable but initially arranged in a circular layout (c.f. Figure 5). 


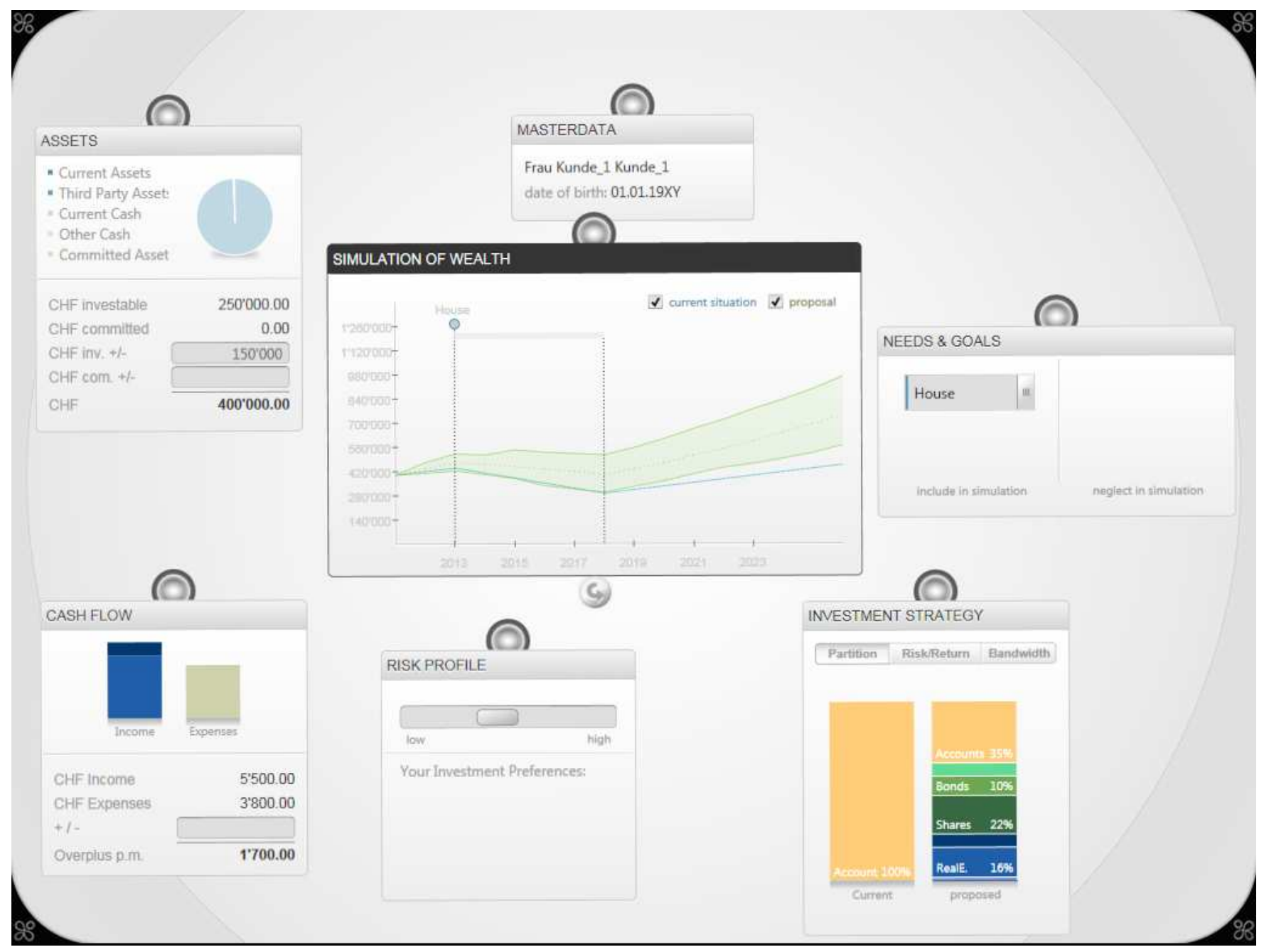

Figure 5: "Zoom and filter" metaphor of Prototype 2 (original interface was in German language).

Switching between artifact work space and relationship building space:

To enable easy switching between the spaces, we propose optimizing the seating position. This principle therefore addresses the environmental aspects rather than the artifact itself. The benefits of adjacent seating arrangements are described in section 2.3 in detail.

Design-Principle 3 (to address generic requirements 3 and 5): Place the participants on adjacent sides around the table so that the RBS and AWS are reachable with minimal body and head movement.

Implementation: For the second evaluation, we raised the table by $15 \mathrm{~cm}$ to a comfortable height of approximately 70 centimeters. This allowed the participants to sit in a slightly tilted, diagonal position and use the table as an arm rest. That seating position allowed participants to focus on the artifact while perceiving the other person's face in their peripheral vision (Figure 4). To switch the space in this arrangement, it is enough to move the eyes only, instead of the head or the whole body.
To make the interface more stable than the slide metaphor style of Prototype 1 we propose using abstraction layers where possible. Abstraction layers also help to reduce the visual complexity of the interface without restricting its functional complexity.

Design-Principle 4 (to address generic requirement 2 and 4): Always display a complete picture of the situation at all times. Design for a stable UI and restrict visualization changes to portions of the screen only.

Implementation: The UI of Prototype 2 was designed with a metaphor of "zoom and filter" [20,29], where only portions of the screen altered at any time. The interface consisted of single widgets, each addressing one activity. Each widget could extend the level of detail when needed (implemented as seen in Figure 5). With this design, only one widget could change its representation at any time, thus providing a mostly stable screen. 


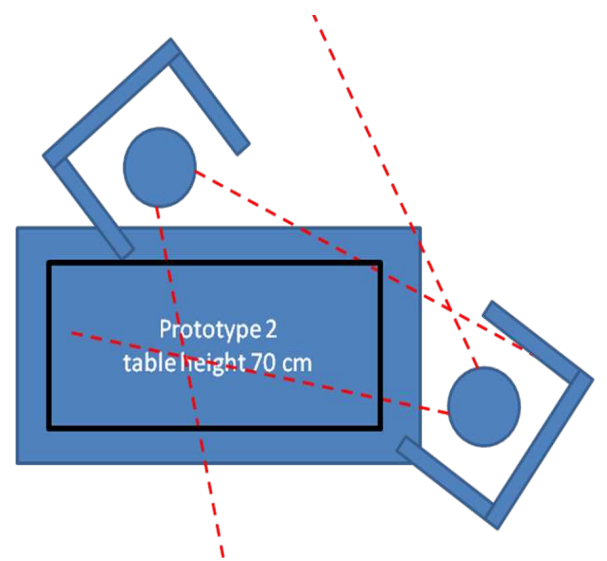

Figure 4: Seating position with Prototype 2 (diagonal seating)

\section{EVALUATION OF PROTOTYPE 2}

Prototype 2 was evaluated like the first one. The experimental evaluation involved 24 clients and 12 advisors in a within-subject design to compare the IT-supported encounter with its traditional counterpart (pen and paper) [20]. Half of the participants started with IT-supported advisory service, and the other half started with the traditional setting. Again, participants were interviewed regarding their experience with the two experimental conditions; the IT-supported sessions were videotaped and all sessions were observed by members of the research team.

The strong connection between eye contact and relationship development is reported in the literature [13], and so we use eye contact as an indicator for being in RBS. However, our video recordings did not allow us to determine eye contacts with certainty; accordingly, we refer to these episodes as "face-gazes." However, we assume that most of these facegazes were actually eye contacts.

We conducted a systematic analysis of face-gazing behavior between clients and advisors. Thus, we opted to encode the viewing directions of both the advisors and clients.

All videos were manually encoded by two observers pressing buttons, each button representing one of the current viewing directions for each participant. This procedure was conducted for the IT-supported sessions of Prototype 2 as well as for the IT-supported sessions of Prototype 1. Each IT-supported test session was videorecorded during the evaluations with both faces and the artifact visible in the recording. We analyzed the 12 sessions of Prototype 1 and 12 sessions of the 24 recordings of Prototype 2 in order to have equal sample size.

The three distinct viewing-directions for each participant were "Focus on the other person" (person looking at the face of the other participant), "Focus on artifact" (person is looking at the artifact), and "Focus elsewhere" (person is looking in any other direction (c.f. Figure 6).

We sampled each recording for 20 second intervals out of every minute of video (second 0-20s, 60-80s, 120-140s, etc.). We expected the small-talk phase to have a lot of face-gazing by nature. We thus marked the point when the small talk phase was left and the participants started to work with the artifact.

\section{$7 \quad$ RESULTS AND DISCUSSION}

In this section, we first report the results of the interviews followed by the results of the face-gazing analysis. Subsequently, we describe the observed effects of our design guidelines.

Interviews: In contrast to the interviews that were conducted with the participants of Prototype 1, participants of the second evaluation hardly reported any problems with respect to the relationship between advisor and client. Only four out of 24 persons briefly mentioned this aspect (In Prototype 110 out of 12 clients mentioned this aspect).

Face-Gazing: Observing the session as a whole, the data show that sessions with Prototype 2 had nearly twice as many face-gazes or eye contacts as did sessions with Prototype 1. Figure 6 shows a state model of the two participants regarding their viewing directions. Face-gazing or eye contact was encoded when both participants looked at each other at the same point in time.

With Prototype 2, participants had an average mutual facegazing ratio of $19.58 \%$ of the time, while they only had $10.75 \%$ with Prototype 1 (Figure 7). The difference is significant (two-sided t-test, $\mathrm{T}(22)=3.23, \mathrm{p}=<.01$ ).

The difference in the face-gazing ratio could not simply be explained by the presence of the dedicated small talk phase. If excluding the small-talk phase from the sample, the length of the gazes was $17.08 \%$ for Prototype 2 and $9.33 \%$ for Prototype 1 (Figure 8), but still significantly different (two-sided t-test, $\mathrm{T}(22)=2.93, \mathrm{p}=<.01$ ).

In addition to this, we analyzed the absolute length of the mutual face-gazing episodes. This analysis revealed that there are more occurrences of longer length face-gazing contacts (10 seconds and more) for Prototype 2 .

An interesting side aspect is that the data show that for both Prototype 1 and 2 the advisors in general focused on the clients more often than clients focusing on advisors. They spent over $30 \%$ of the session's time looking towards the clients. 

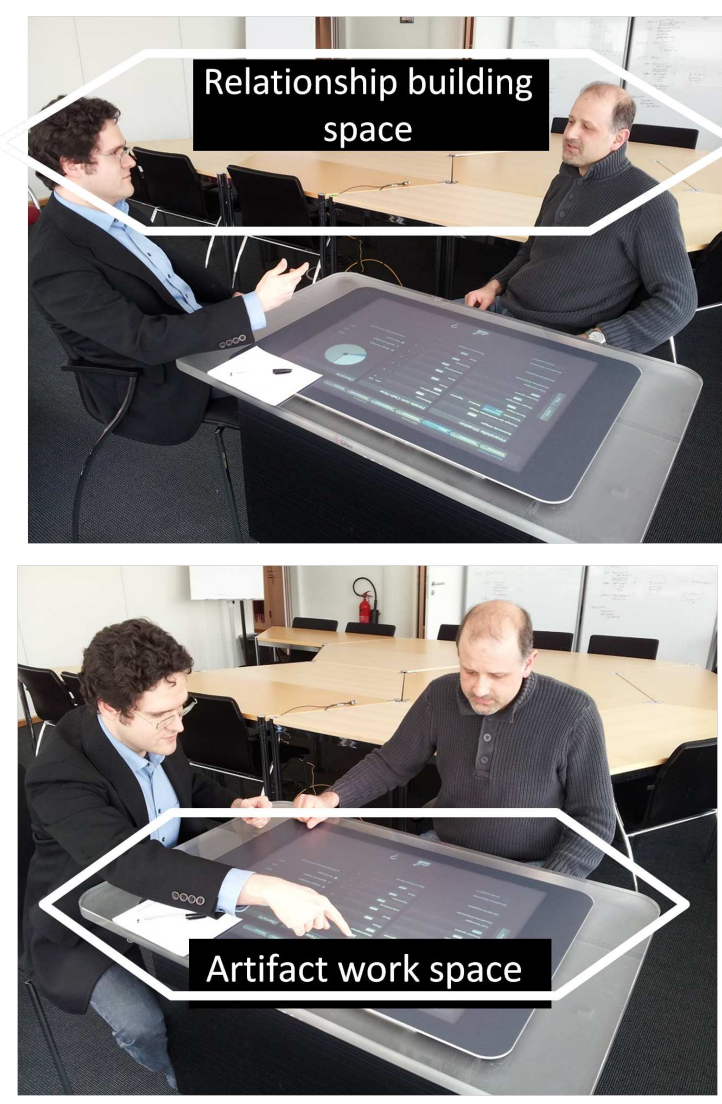

Figure 6: States of view when working on the artifact.

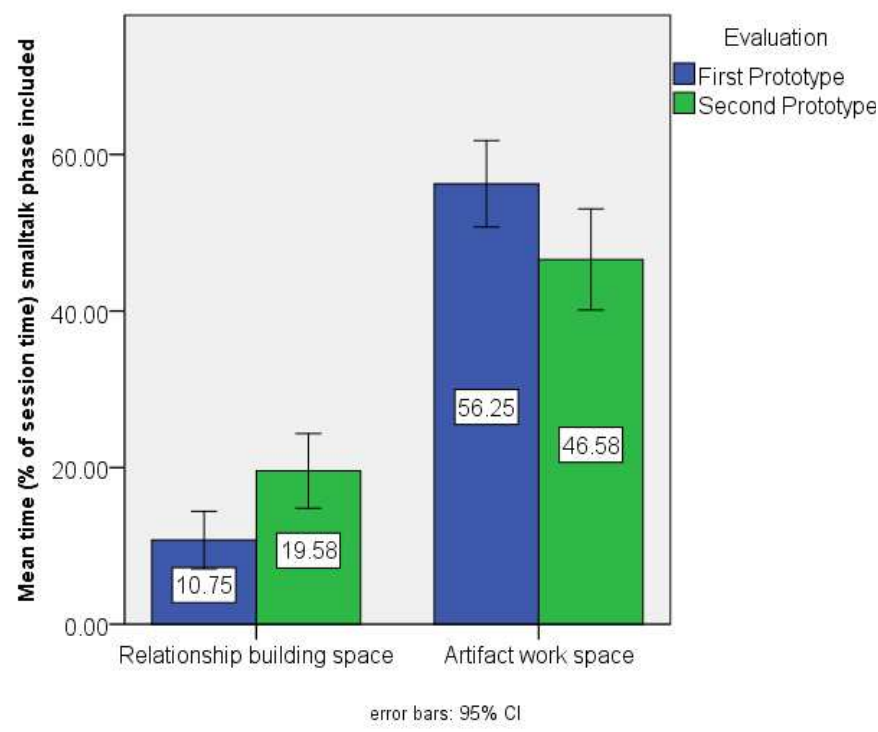

Figure 7: Mean time in the relationship building space with the small-talk phase included.

In greater detail, for Prototype 1 the advisors looked an average of $31.58 \%$ of the time towards the client and the client looked $16.83 \%$ of the time towards the advisors. For Prototype 2 the numbers were $38.08 \%$ for the advisor and $27.83 \%$ for the client. The difference for the client is significant (two-sided t-test, $\mathrm{T}(22)=3.38, \mathrm{p}<=0.01$ ). Without the small talk phase the average face-gazing time was $14.6 \%$ vs. $23.6 \%$ for the client (two-sided t-test, $\mathrm{T}(22)=2.79, \mathrm{p}<=0.05)$ and $30.5 \%$ vs. $36.5 \%$ for the advisors.

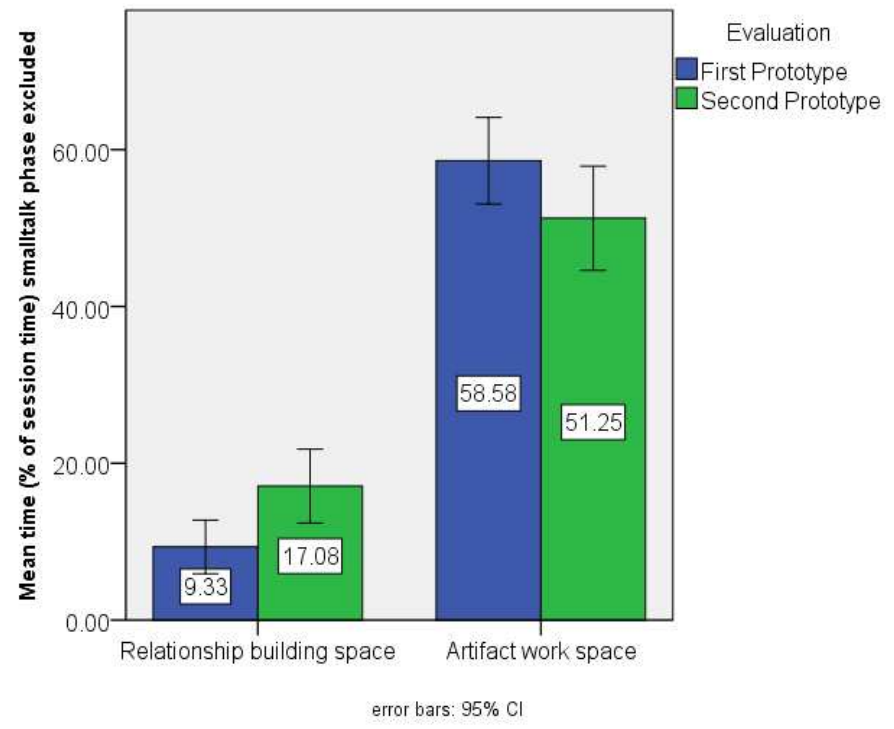

Figure 8: Mean time in the relationship building space without the small-talk phase.

Creating and maintaining the relationship building space:

The idea of having a dedicated small-talk phase at the beginning of the advisory endeavor was adopted well by the advisors. All sessions with Prototype 2 had an extensive small-talk phase (average of 10 minutes) between client and advisor prior to interacting with the artifact.

During that phase we could see a high degree of mutual face-gazing, supporting the assumption that mutual facegazing moments are desired by both parties in such encounters. The additional dedicated small talk phase alone increased the overall amount of successful mutual facegazing in comparison to that of the first evaluation. But even when subtracting these episodes from the face-gazing analysis, the situation for Prototype 2 still contained significantly more mutual face-gazing when compared to Prototype 1.

\section{Switching between artifact work space and relationship building space:}

The observation of the trials as well as the video recordings revealed the following aspects. The changes in table height had a strong effect on the predominant seating position. Due to the fact that the table was not square and people were seated relatively close to the artifact (in a 90 degree position), it was not possible for customers to focus on the artifact and simultaneously to have the advisor's face in 
their peripheral view. To gaze at the advisor's face, they had to leave the artifact focus and rotate their heads towards the advisor.

Now, with the rotated seating position of the second evaluation, customers had the face of the advisor and the artifact in nearly the same line of sight. Without the need to rotate their heads, they could sense when the advisor was looking at them and make direct eye contact when they felt it was appropriate.

This altered seating position, we believe, resulting from the change in table height, allowed customers to use the table as an armrest. Apparently, people prefer using a table as an armrest in such situations, as we also observed during the unsupported (pen and paper traditional style) advisory encounters.

These situations are sensitive to minute changes in the physical environment (like raising the table height by 15 $\mathrm{cm}$ ), and the effects can be manifested in the overall impression of the participants.

We assume that the reduced cognitive effort on the part of the advisor as well as that of the client leads to increased chances that eye contact can be established. This is supported by our face-gazing data indicating that the advisor looks more often at the client in the Prototype 2 condition compared to in the Prototype 1 condition. This effect is even stronger for the clients. In the Prototype 2 condition the clients look considerably longer at the face of the advisor compared to in the Prototype 1 condition.

However, we mainly attribute the altered UI for the change in cognitive effort on the client side and the extended training period for the change of load on the advisor's side solely by argument. Since the experiment did not control for these variables separately, we cannot be certain.

\section{LIMITATIONS}

The analysis we conducted on our experimental scenarios did not allow us to control for all possible variables. Thus, we cannot be certain of the amount of influence of the different variables discussed or the directions of the influences. Our in-depth analysis, however, provides a rich understanding of the situation that would not have been possible in tightly controlled experimental settings.

The experiments were conducted in the Swiss financial sector, and their direct applicability is limited to this sector. We are confident it can be generalized in two directions: The results should be applicable to other Western countries, as underlying issues of principal agency conflicts and trust building are common there too. We believe they can also be generalized to other collaborative settings where establishing and maintaining personal relationships is crucial. Primary candidates are other sales oriented service encounters, e.g., in insurances or travel agencies. Conflict resolution meeting, consensus finding sessions or negotiations may also benefit from our results.

\section{CONCLUSION}

In this paper we described the severe effects of insufficient relationship building when working with IT-artifacts in advisory encounter situations. Based on observations and existing literature, we derived five generic requirements and four design principles that specifically address requirements for successful relationship building. These principles address the design of the artifact itself as well as the environment and process. In a subsequent evaluation with an enhanced prototype, following these guidelines, we found the effects of insufficient relationship building being reduced to the point of not being reported any longer by the majority of the test participants. Therefore, we conclude that systems following these guidelines should be able to maintain the level of relationship building compared to that of traditional settings.

In contrast to related work, this paper not only addresses the physical environment but also focuses on UI design issues related to relationship building. In the interviews we also found evidence that a suboptimal design of the IT-artifact can directly hamper relationship building.

Our contribution should help in the development of future collaboratively used IT-artifacts, thus directly contributing to the research stream of IT supported face-to-face collaboration [8].

By addressing the relationship building issue, we also enable practitioners in the finance industry to address a crucial aspect of their value proposition, i.e. a trusting relationship to their clients.

In this contribution, we focus on advisory scenarios, where the customer visits the advisor in her office because we believe that these settings have interesting characteristics from a scientific perspective as well as an increasing importance from a practitioner's perspective. From a research perspective, we helped understanding relationship building while working with IT artifacts in service encounters. For practitioners on the other hand, this contribution could be meaningful to design future systems without hampering the very fragile aspect of relationship building. Advisory scenarios are the central communication channels for several core disciplines in our current service economy, including medicine, law, finance and many sales processes. Advisory settings are always used when the information that is communicated is complex and/or very relevant to the perceiving person. This can mean that high amounts of money are involved as in financial situations or that the risks of certain procedures are very high such as in medicine or in legal advice. 
It is therefore very important that the client is well informed to make an informed decision. This includes transferring the information as such, but it also includes the creation of a trustful relationship because the lack of such an relationship will also hamper the cooperation and the exchange of knowledge and advice (e.g., if persons do not ask relevant questions or conceal important information).

Thus, these kinds of processes are omnipresent in our current economic, medical and legal system with severe implications for the advised persons but possibly also for the advisor in case the advisor process fails. We believe that due to the awareness of the sensitivity of the process, the introduction of IT into these processes has hardly been undertaken.

The complexity of the transferred information, however, as well the challenging learning process that the participants of the advisory process have to go through, makes the introduction of IT very promising for the following reasons: The process of the advisory session can be supported in such a way that no central information aspects are omitted. Core aspects of the knowledge that has to be transmitted can be visualized and animated to improve the understanding of the complex subjects. Finally, the discussed topics can be documented and used to recall complex content after the end of the advisory session.

For these reasons, we expect an increased use of IT in such sensitive areas. These new opportunities, however, also impose new challenges for the design of advisory support systems. If we want to realize the benefits, we have to understand how these kinds of systems impact the relationship building between participants and be aware of the implications for the design. This article seeks to make a contribution in this direction.

\section{ACKNOWLEDGEMENTS}

The software was developed as part of the CTI project 'Banking Advisory 2.0' funded by the Swiss Federal Government. This was a group effort. We are grateful for the significant contribution of Philipp Nussbaumer, Inu Matter and Didier Bertschinger in designing the system and collecting evaluation data. We furthermore acknowledge the great support of Gerhard Buurmann (SDFB*), Anna Lang, Martin Scherrer and David Zysset in making this project a success.

* SDFB: swiss design institute for finance and banking
1. Ballendat, T., Marquardt, N., and Greenberg, S. Proxemic interaction: designing for a proximity and orientation-aware environment. ACM International Conference on Interactive Tabletops and Surfaces, ACM (2010), 121-130.

2. Bonnard, Q., Jermann, P., Legge, A., Kaplan, F., and Dillenbourg, P. Tangible paper interfaces: interpreting pupils' manipulations. Proceedings of the 2012 ACM international conference on Interactive tabletops and surfaces, ACM (2012), 133-142.

3. Clark, H.H. and Brennan, S.E. Grounding in communication. Perspectives on socially shared cognition 13, APA (1991), 127-149.

4. Cohn, R.C. Von der Psychoanalyse zur themenzentrierten Interaktion: Von der Behandlung einzelner zu einer Pädagogik für alle. Klett-Cotta, (2000).

5. Crosby, L.A., Evans, K.R., and Cowles, D. Relationship Quality in Services Selling: An Interpersonal Influence Perspective. Journal of Marketing 54, (1990), 68-81.

6. Ellis, C.A., Gibbs, S.J., and Rein, G. Groupware: some issues and experiences. Communications of the ACM 34, (1991), 39-58.

7. Hall, E.T. The hidden dimension. Doubleday, 1966.

8. Haller, M., Billinghurst, M., Leithinger, J., Leitner, D., and Seifried, T. Coeno: enhancing face-to-face collaboration. Proceedings of the 2005 international conference on Augmented tele-existence, ACM (2005), 40-47.

9. Heinrich, P., Giesbrecht, T., Kilic, M., and Schwabe, G. Situated Learning in Bankberatungen. Bonner Köllen Verlag (2012), 261-266.

10. Herrington, J. and Oliver, R. Critical characteristics of situated learning: Implications for the instructional design of multimedia. Learning with technology, proceedings of ASCILITE'95, (1995), 235-262.

11. Inkpen, K.M., Hancock, M.S., Mandryk, R.L., and Scott, S.D. Collaboration around a tabletop display: Supporting interpersonal interactions. tech report, Simon Fraser University, (2002).

12. Jungermann, H. Advice giving and taking. Proceedings of the 32nd Annual Hawaii International Conference on System Sciences, 1999. HICSS-32, IEEE (1999).

13. Kleinke, C.L. Gaze and eye contact: a research review. Psychological bulletin 100, 1 (1986), 78-100. 
14. Kruger, R., Carpendale, S., Scott, S.D., and Greenberg, S. How people use orientation on tables: comprehension, coordination and communication. Proceedings of the 2003 international ACM SIGGROUP conference on Supporting group work, (2003), 369-378.

15. Lewe, H. and Krcmar, H. The design process for a computer-supported cooperative work research laboratory: the Hohenheim CATeam room. Journal of Management Information Systems, (1991), 69-85.

16. Mantei, M. Capturing the capture concepts: a case study in the design of computer-supported meeting environments. Proceedings of the 1988 ACM conference on Computer-supported cooperative work, ACM (1988), 257-270.

17. Matsushita, M., Shirai, Y., Oguro, T., et al. A Face-toface Collaboration Support System that Optimizes Direction of Projected Information to Each Stakeholder. Transactions 46, 7 (2005), 1603-1617.

18. Nguyen, D. and Canny, J. MultiView: spatially faithful group video conferencing. Proceedings of the SIGCHI Conference on Human Factors in Computing Systems, ACM (2005), 799-808.

19. Novak, J. Mine, yours... ours? Designing for principalagent collaboration in interactive value creation. Wirtschaftsinformatik 2009, Vienna, (2009).

20. Nussbaumer, P., Matter, I., and Schwabe, G. "Enforced" vs. "Casual" Transparency - Findings from IT-Supported Financial Advisory Encounters. ACM Transactions on Management Information Systems, (2012), Article 11.

21. Nussbaumer, P. and Matter, I.S. What You See Is What You (Can) Get? Designing for Process Transparency in Financial Advisory Encounters. Interact 2011, (2011), 277-294.

22. Papadopoulou, P., Andreou, A., Kanellis, P., and Martakos, D. Trust and relationship building in electronic commerce. Internet Research 11, 4 (2001), 322-332.

23. Prahalad, C.K. and Ramaswamy, V. Co-creation experiences: The next practice in value creation. Journal of interactive marketing 18, 3 (2004), 5-14.

24. Rogers, Y. and Lindley, S. Collaborating around vertical and horizontal large interactive displays: which way is best? Interacting with Computers 16, 6 (2004), 1133-1152.
25. Schmidt-Rauch, S. and Nussbaumer, P. Putting Value Co-Creation into Practice: A Case for Advisory Support. Proceedings of the 19th European Conference on Information Systems 2011, (2011), Paper 138.

26. Schwabe, G. and Nussbaumer, P. Why IT is not being used for financial advisory. Proceedings of the 17th European Conference on Information Systems 2009, (2009), Paper 450.

27. Scott, S.D., Grant, K.D., and Mandryk, R.L. System guidelines for co-located, collaborative work on a tabletop display. ECSCW 2003, (2003), 159-178.

28. Shaer, O., Strait, M., Valdes, C., Feng, T., Lintz, M., and Wang, H. Enhancing genomic learning through tabletop interaction. Proceedings of the 2011 annual conference on Human factors in computing systems, ACM (2011), 2817-2826.

29. Shneiderman, B. The eyes have it: a task by data type taxonomy for information visualizations. Visual Languages, 1996. Proceedings., IEEE Symposium on, (1996), $336-343$.

30. Sommer, R. Personal Space. The Behavioral Basis of Design, Prentice-Hall, (1969).

31. Steimle, J., Khalilbeigi, M., Mühlhäuser, M., and Hollan, J.D. Physical and digital media usage patterns on interactive tabletop surfaces. ACM International Conference on Interactive Tabletops and Surfaces, ACM (2010), 167-176.

32. Stewart, J., Bederson, B.B., and Druin, A. Single display groupware: a model for co-present collaboration. Proceedings of the SIGCHI conference on Human factors in computing systems: the CHI is the limit, (1999), 286-293.

33. Tse, E., Greenberg, S., Shen, C., and Forlines, C. Multimodal multiplayer tabletop gaming. Computers in Entertainment (CIE) 5, (2007), Article No. 12.

34. Wallace, J.R. and Scott, S.D. Contextual design considerations for co-located, collaborative tables. 3rd IEEE International Workshop on Horizontal Interactive Human Computer Systems, 2008. TABLETOP 2008, (2008), 57-64.

35. Weber, W.G. Organizational conditions fostering prosocial work orientations in teams. Innovative Theories, Tools, and Practices in Work and Organizational Psychology. Seattle, WA: Hogrefe and Huber, (2000), 75-96. 